\title{
Hemorrhagic Bullous Angina: A Case Report and Review of the Literature
}

\author{
Case Report $>\begin{aligned} & \text { Sara Rodrigues, Marielle Albrechete, Rafael Monsanto, Azis Chagury, Fabio Lorenzetti } \\ & \text { Department of Otolaryngology, Banco de Olhos de Sorocaba Hospital, Sorocaba, Brazil }\end{aligned}$
}

Abstract

Hemorrhagic bullous angina (HBA) is described as the sudden onset of one or more bullous lesions in the oral cavity, not attributable to other vesiculobullous diseases, blood dyscrasias, or autoimmune and vascular diseases. These lesions occur almost exclusively in the oral cavity, particularly in the soft palate, and do not affect the masticatory mucosa. Here we present the case of a 57-year-old male who had

\section{Introduction}

Hemorrhagic bullous angina (HBA) is defined as the sudden onset of one or more bullous lesions in the oral cavity, not attributable to other vesiculobullous diseases, blood dyscrasias, or autoimmune and vascular diseases (1-4). Local recurrences are frequent and usually occur at the same site as previous lesions. These lesions occur almost exclusively in the oral cavity, particularly in the soft palate, and do not affect the masticatory mucosa $(1,2)$. HBA is a rare condition, with only around 130 reported cases.

Here we aim to report a case of HBA, focusing on clinical aspects, differential diagnosis, and treatment, along with a review of the literature on the topic.

This study was presented at the $45^{\text {th }}$ Brazilian Otorhinolaryngology Meeting, 25-28 November 2015, Fortaeza, Brazil.

Address for Correspondence: Marielle Albrechete

E-mail: marialbrechete@hotmail.com

Received Date: 28.06.2016 Accepted Date: 11.08 .2016

(c) Copyright 2016 by Official Journal of the Turkish Society of Otorhinolaryngology and Head and Neck Surgery Available online at www.turkarchotorhinolaryngol.org DOI: 10.5152/ta0.2016.1795

\section{Case Report}

This case report has been approved by the Ethics Committee Board of our hospital, and the patient has agreed with the publication of clinical information and pictures, as stated in a signed informed consent form.

A 57-year-old male complained of bulging in the soft palate, which he described as "a balloon a spontaneously ruptured hemorrhagic bulging in his soft palate diagnosed as HBA, along with discussion of the literature. In conclusion, HBA is a rare, benign, oral disease with low complication rates. Diagnosis is essentially clinical, and treatment consists of local hygiene and prevention of oral trauma.

Keywords: Bullous lesion, oral hemorrhage, vesiculobullous skin diseases that filled up his mouth," appearing 2 hours before his visit. The lesion had increased in size within a few seconds, and then, its volume decreased within a few minutes. He denied oral trauma, bleeding, and dyspnea. The patient had diabetes mellitus and hypertension. He had no history of hematological disease, bleeding disorder, and previous surgery. He also did not have a familial history of bleeding disorders. He was not a smoker, and he did not consume alcohol.

In oral cavity examination, there was a midline subepithelial bullous injury located between the posterior border of the hard palate and the uvula (Figure 1). Findings of rhinoscopy and otoscopy were normal. The capillary blood glucose level at presentation was $336 \mathrm{mg} / \mathrm{dL}$ and decreased to $198 \mathrm{mg} / \mathrm{dL}$ after subcutaneous application of 6 IU of regular insulin and intravenous administration of $250 \mathrm{~mL}$ of $0.9 \%$ sodium chloride (saline solution). Findings of blood tests, including hemogram (including platelet count), coagulation tests, and serum nitrogen, creatinine, aspartate aminotransferase (AST), and alanine aminotransferase (ALT) tests, were normal. 
Hemorrhagic bullous angina was suspected on the basis of the clinical history and physical examination findings. The patient was empirically prescribed crushed tranexamic acid (Lysteda, Ferris Pharmaceutical; Parsippany, USA) orally (500 mg, every $8 \mathrm{~h}$, for 5 days). He was also advised to avoid hot and solid, crispy food and to prefer liquid or semisolid meals. The patient was decided to be followed as an outpatient, and a new appointment was scheduled after 3 days for follow-up.

The follow-up period was uneventful, and the patient was asymptomatic after 3 days. Oral examination revealed a small lesion in the soft palate mucosa without any bulging or signs of bleeding (Figure 2). After 7 days, findings of oral cavity examination were unremarkable, and the patient was advised to return in case of symptom recurrence.

\section{Discussion}

Hemorrhagic bullous angina lesions occur after oral trauma, mainly after the ingestion of hot and crispy food; however, sneezing and routine dental procedures have also been reported to cause HBA (2-5). Potential factors involved include the use of inhaled corticosteroids and arterial hypertension (1-5). Grinspan et al. (1) have also reported a $44.4 \%$ incidence of hyperglycemia or diabetes in patients with HBA. Symptoms include stabbing sensation, followed by bulging in the soft palate (4-9). The lesions often reach over 3 or $4 \mathrm{~cm}$ within a few minutes and cause the patients to seek emergency care because of a fear of choking (9). If the lesion ruptures, the bloody content of the lesion flows into the oral cavity; these bleedings are usually self-limited, ceasing after a few minutes (1-10). Nonetheless, one study has reported the case of a patient who needed tracheostomy due to upper airway obstruction by massive bleeding after the rupture of HBA (9).

Histopathological examination of biopsy samples suggests that the cleavage plane of the bullae is within the basal membrane; however, some debris may be present on the top of the blister (11). Previous studies demonstrated that analysis of such specimens (stained with hematoxylin and eosin) does not demonstrate epithelial atrophy; however, samples stained by silver stain show decreased number of elastic fibers $(10,11)$. These data suggest that a lack of support to the small blood vessels in the areas surrounding HBA increases the chances of the development of bleeding in case of trauma. Direct immunofluorescence of the lesion is usually negative, although some authors have demonstrated the presence of inflammatory mediators such as complement component 3 (C3) and immunoglobulin $\mathrm{G}(\mathrm{IgG})$ (7-11).

Hemorrhagic bullous angina should be distinguished from other subepithelial bullous lesions involving the oral cavity. Some possible differential diagnoses include mucosal pemphigoid, cicatricial pemphigoid, oral amyloidosis, epidermolysis bullosa acquisita, erythema multiforme, fixed drug eruption, linear IgA dermatosis, and

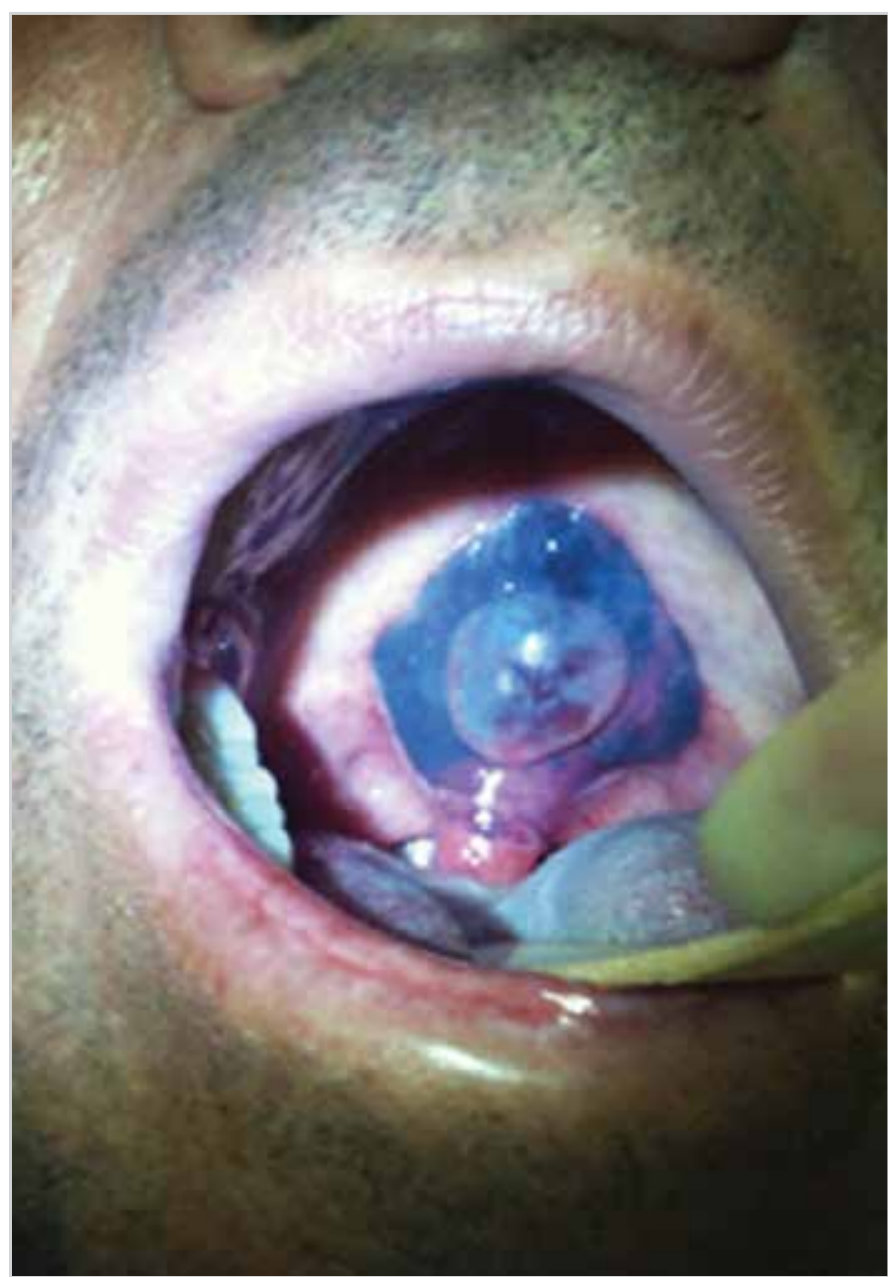

Figure 1. Oral cavity examination showing a single subepithelial bullous lesion lying over an adjacent area of ecchymosis on the soft palate

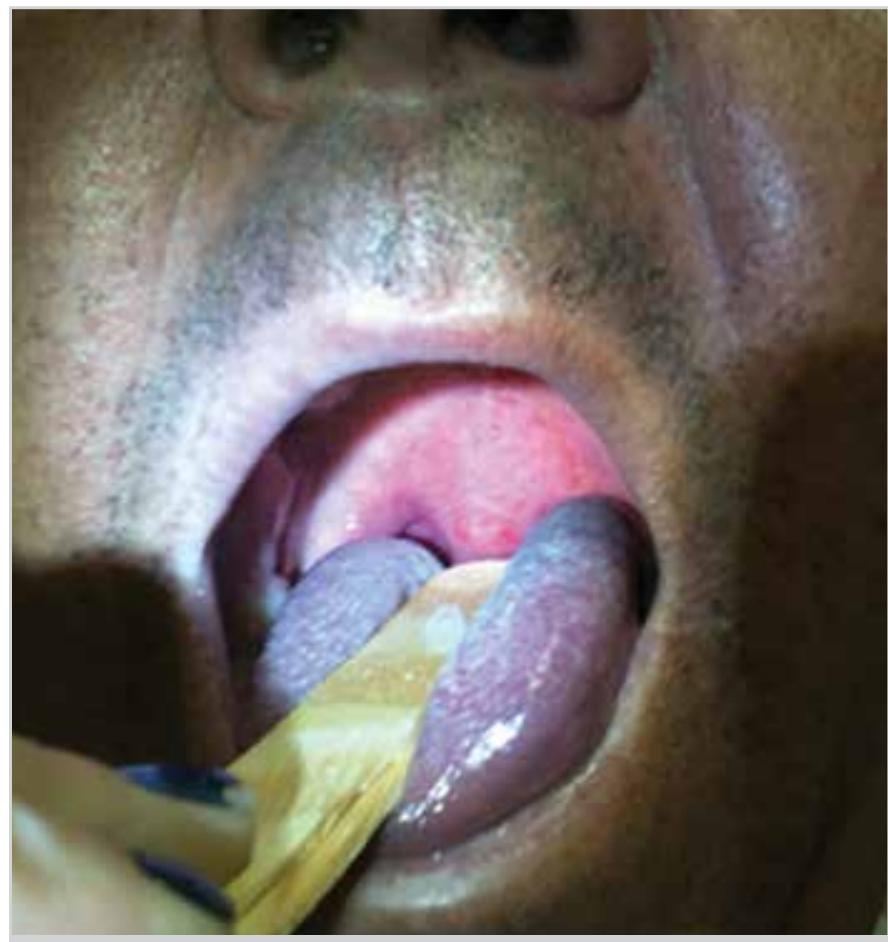

Figure 2. Follow-up examination demonstrating a small vesicular lesion in the base of the uvula; the bullous lesion was absent after 3 days of the initial symptoms 
bullous lichen planus $(12,13)$. Anamnesis, clinical examination, and histopathology and immunofluorescence are usually sufficient to enable accurate diagnosis (3-10). HBA is not associated with skin lesions; the oral lesions are hemorrhagic, do not leave any residual scarring, and are not associated with desquamative gingivitis. In addition, findings of histopathological and immunofluorescence analysis are frequently unremarkable (3-10). Furthermore, findings of blood tests (including hemogram, platelet count, and coagulation tests) are usually normal. In contrast, the oral cavity lesions of mucosal pemphigoid may or may not be filled with blood. In addition, they are often associated with scarring and desquamative gingivitis (4). The cicatricial pemphigoid presents with a non-hemorrhagic oral blister that leaves a visible scar; the lesions are associated with desquamative gingivitis and systemic mucosal involvement (including nasal, esophageal, laryngeal, and vaginal mucosa) $(4,12,13)$. The bullae in oral amyloidosis are small, multiple, and persistent. Moreover, there is skin involvement and macroglossia. Diagnosis of this entity can be made with histopathological analysis of biopsy samples stained with Congo red (12). The oral lesions of the epidermolysis bullosa are painful and do not occur without skin involvement (10-13). Furthermore, epidermolysis bullosa is caused by autoantibodies to Type IV collagen, and these autoantibodies are absent in HBA (10-13). Erythema multiforme usually presents with characteristic "target" skin lesions as well as multiple erosions and crusts on the lips (4). Fixed drug eruption occurs after the use of a certain medication, and the lesions evolve from erythematous macules to edematous plaques. Later, they can develop into bullae with crusting, which heal after a few days, leaving hyperpigmentation. Furthermore, morbilliform, scarlatiniform, or multiforme skin lesions are common (14). Although the lesions caused by linear $\operatorname{IgA}$ disease may be similar to HBA, histopathologic analysis is usually enough to perform a differential diagnosis between these two diseases - while linear IgA disease lesions have neutrophilic infiltrate and the basement membrane shows linear impregnation of the basement membrane under immunofluorescence, HBA lesions do not have inflammatory infiltrate or changes in the basement membrane (12). Immunofluorescence of oral bullous lichen planus biopsy samples also show linear deposits of fibrinogen along the basement membrane zone (4).

No specific treatment for HBA is required, although large lesions causing respiratory distress should be incised with a scalpel for drainage. When they rupture, the bleeding is self-limited; however, airway patency needs to be secured. Complete remission of the lesions is expected within 10 days (1-10). It is recommended to use anti-inflammatory or antibacterial solutions as mouthwash ( $2 \%$ chlortetracycline or $0.25 \%$ chlorhexidine gluconate) 2 to 3 times a day for 3 days to prevent secondary infection at the site of the lesion $(1,4,10)$.
We report a typical case of HBA (after excluding other possible causes), as demonstrated with pictures showing the morphological characteristics of the lesion. Furthermore, we describe in detail the follow-up and course of the disease. Our review of the literature provides a more comprehensive approach to the pathology, providing an updated basis for explaining the pathophysiological mechanisms, possible etiologies, and diagnosis and treatment options than those provided in previous published studies on HBA $(1-5,7,8$, 10-14).

\section{Conclusion}

Hemorrhagic bullous angina is a rare, benign, oral disease, with low complication rates. Diagnosis is essentially clini$\mathrm{cal}$, and treatment consists of local hygiene and the prevention of oral trauma.

Informed Consent: Written informed consent was obtained from patient who participated in this study.

Peer-review: Externally peer-reviewed.

Author contributions: Concept - S.R., M.A., A.C.; Design - S.R., M.A.; Supervision - R.M., A.C., F.L.; Resource - S.R., M.A.; Materials - S.R., M.A., A.C.; Data Collection and/or Processing - S.R., M.A., R.M.; Analysis and/or Interpretation - R.M., A.C., F.L.; Literature Search - S.R., R.M.; Writing - S.R., M.A., R.M.; Critical Reviews - R.M., A.C., F.L.

Conflict of Interest: No conflict of interest was declared by the authors.

Financial Disclosure: The authors declared that this study has received no financial support.

\section{References}

1. Grinspan D, Abulafia J, Lanfranchi H. Angina bullosa hemorrhagica. Int J Dermatol 1999; 38: 525-8. [CrossRef]

2. Horie N, Kawano R, Inaba J, Numa T, Kato T, Nasu D, et al. Angina bullosa hemorrhagica of the soft palate: a clinical study of 16 cases. J Oral Sci 2008; 50: 33-6. [CrossRef]

3. Beguerie JR, Gonzalez S. Angina bullosa hemorrhagica: report of 11 cases. Dermatol Reports 2014; 12: 5282. [CrossRef]

4. Yamamoto K, Fujimoto M, Inoue M, Maeda M, Yamakawa N, Kirita T. Angina bullosa hemorrhagica of the soft palate: report of 11 cases and literature review. J Oral Maxillofac Surg 2006; 64: 1433-6. [CrossRef]

5. Giuliani M, Favia GF, Lajolo C, Miani CM. Angina bullosa haemorrhagica: presentation of eight new cases and a review of the literature. Oral Dis 2002; 8: 54-8. [CrossRef]

6. Higgins EM, du Vivier AW. Angina bullosa haemorrhagica--a possible relation to steroid inhalers. Clin Exp Dermatol 1991; 16: 244-6. [CrossRef]

7. Volkweis MR, Galeazzi S. Angina bolhosa hemorragica: uma revisao de 14 casos. RFO UPF 2010; 15: 298-301.

8. Stephenson P, Lamey PJ, Scully C, Prime SS. Angina bullosa haemorrhagica: clinical and laboratory features in 30 patients. Oral Surg Oral Med Oral Pathol 1987; 63: 560-5. [CrossRef] 
9. Pahl C, Yarrow S, Steventon N, Saeed NR, Dyar O. Angina bullosa haemorrhagica presenting as acute upper airway obstruction. Br J Anaesth 2004; 92: 283-6. [CrossRef]

10. Sasai IF, Fujiyama T. Histological study on the pathogenesis of epidermolysis bullosa acquisita. Tohoku J Exp Med 1969; 99: 9-17. [CrossRef]

11. Kurban M, Kibbi AG, Ghosn S. Expanding the histologic spectrum of angina bullosa hemorrhagica: report of one case. Am J Dermatopathol 2007; 29: 477-9. [CrossRef]
12. Curran AE, Rives RW. Angina bullosa hemorrhagica: an unusual problem following periodontal therapy. J Periodontol 2000; 71: 1770-3. [CrossRef]

13. Forman L, Nally FF. Oral non-dystrophic bullous eruption mainly limited to the gingivae: a mechano bullous response. A variant of cicatricial mucous membrane pemphigoid? $\mathrm{Br} \mathrm{J}$ Dermatol 1977; 96: 111-7. [CrossRef]

14. Sehgal VN, Gangwani OP. Fixed drug eruption. Current concepts. Int J Dermatol 1987; 26: 67-74. [CrossRef] 